\title{
All-Optical Reservoir Computing on a Photonic Chip Using Silicon-Based Ring Resonators
}

\author{
Florian Denis-Le Coarer ${ }^{(}$, Marc Sciamanna, Andrew Katumba ${ }^{\circledR}$, Matthias Freiberger ${ }^{\circledR}$, Joni Dambre ${ }^{(0)}$, \\ Peter Bienstman (i), and Damien Rontani (1)
}

\begin{abstract}
We present in our work numerical results on the performance of a $4 \times 4$ swirl-topology photonic reservoir integrated on a silicon chip. Nonlinear microring resonators are used as nodes. We analyze the performance of such a reservoir on a classical nonlinear Boolean task (the delayed XOR task) for: various designs of the reservoir in terms of lengths of the waveguides between consecutive nodes, and various injection parameters (injected power and optical detuning). From this analysis, we find that this kind of reservoir can perform-for a large variety of parameters-the delayed XOR task at $20 \mathrm{~Gb} / \mathrm{s}$ with bit error rates lower than $10^{-3}$ and an averaged injection power lower than $2.5 \mathrm{~mW}$.
\end{abstract}

Index Terms-Reservoir computing, silicon photonics, ring resonators.

\section{INTRODUCTION}

$\mathbf{T}$ HE development of machine learning solutions in the physical layer appears as a promising approach to address the new challenges brought by the increasing amount of data to process [1]. Compared to existing software-based solutions, dedicated hardware platforms allow to process data at higher speed and better energy efficiency [2], even enabling real-time computation [3]. Amongst the existing machine-learning approaches, reservoir computing - a supervised learning technique that appeared a decade ago - has focused a lot of attention [4]-[6]. This is mainly due to its relatively straightforward implementation, both in software and hardware, and a simple training proce- dure. As a result, this concept has displayed state-of-the-art performance on various hardware platforms [7]-[9], including photonics ([10] and references therein).

Photonics reservoir computing (PRC) is a candidate technology that has attracted lots of attention in the last few years [11] [24], due to its ability to perform typical tasks of artificial neural-networks: emulation of simple boolean operations [23], pattern generation [24], chaotic time series prediction [17], or bit-sequences recognition [20]. Multiple photonic implementations have been proposed and they include a single nonlinear node with delayed feedback such as optoelectronic oscillators [11] and laser diode with optical feedback [12]; coupled photonic crystal cavities [24], integrated photonic reservoirs using passive nodes made of delay lines and splitters [22], [23], networks of semiconductor optical amplifiers [19], or networks of InGaAsP/InP-based ring resonators [20].

In this work, we propose a novel photonics architecture of reservoir computing integrated on a silicon chip, using Silicon-on-Insulator (SOI) microring (MR) resonators as nonlinear nodes. This integrated element exhibits rich nonlinear dynamical behaviors [25]-[31]. SOI microrings resonators are mostly used as optical filters [32], but can also be integrated in more complex architectures and perform other types of alloptical information processing such as boolean functions [33], thresholding [34], pulse restoration [35], or ASK-to-PSK conversion [36].

We build here a $4 \times 4$ swirl reservoir topology using SOI microring resonators as nodes. We perform an in-depth numerical analysis of the performance of such a reservoir and investigate the impact of new degrees of freedom, namely the injected power, the optical detuning, and possible resonance mismatches between the microring resonators. The performance of the reservoir architecture is based on the typical delayed XOR task by quantifying the bit-error rate (BER). We compare the performance of our reservoir with those of a similar topology, but using linear nodes made of waveguides, splitters, and combiners [22], [23]. We demonstrate that our architecture can reach BER level comparable to those of the passive reservoir $\left(<10^{-3}\right)$ at data rate of $20 \mathrm{~Gb} / \mathrm{s}$, and over a wide range of design parameters. Furthermore, the power consumption required to reach this level of performance using Return-to-Zero (RZ) input signal is only $2.4 \mathrm{~mW}(0.15 \mathrm{~mW}$ per node $)$, which is in the same order of magnitude than the power used in the previous design, where Non-Return-to-Zero (NRZ) input signals were necessary to perform at best. 


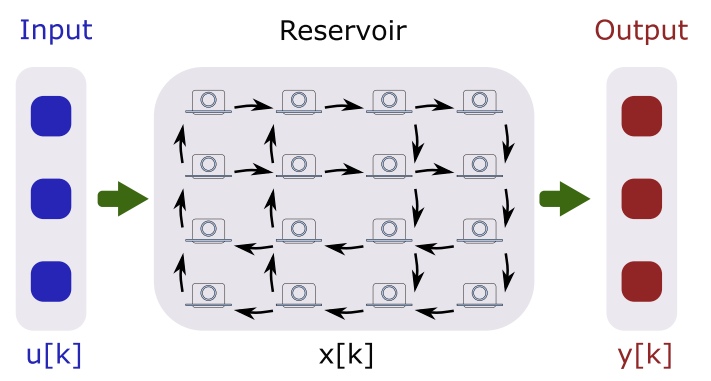

Fig. 1. Illustration the $4 \times 4$ swirl topology of the photonics reservoir under investigation. Each node is a nonlinear microring resonator. Nodes are linked by waveguides with typical losses of $3.0 \mathrm{~dB} / \mathrm{cm}$.

This work is organised as follows. We first describe the theoretical reservoir model used in our numerical simulation, and the physical model of a SOI nonlinear microring resonator, which is the building block of the reservoir. Then after that, from an analysis of the stability of a nonlinear ring resonator, we study the optimum parameters of injection (power injected in each node, and optical detuning) for the reservoir in order to get the best performance. Then we present the simulated performance of the new reservoir architecture studied in this work. Finally, a last section is devoted to discussions and conclusions.

\section{RESERVOIR MODEL}

The photonic reservoir is a 16 -node $(4 \times 4)$ swirl reservoir in which each node is a nonlinear microring resonator (see [25], [26] and the following section). The swirl topology - introduced in [22] and [23] - allows sufficient mixing of the input signals while satisfying to the planarity constraint of an integrated implementation and minimizing the power losses in the structure at each combiner. The connections between neighbouring nodes are ensured by long waveguides, which introduce a non-negligible inter-delay due to the finite-time propagation of optical signals. We depict in Fig. 1 a schematic of the integrated photonic reservoir studied in this work.

The reservoir model is given by (1)-(2):

$$
\begin{aligned}
\mathbf{x}[k+1] & =f\left(\mathbf{x}[k], \mathbf{W}_{\text {res }} \mathbf{x}[k]+\mathbf{W}_{i n}\left(\mathbf{u}[k+1]+u_{\text {bias }}\right)\right), \\
\mathbf{y}_{\text {out }}[k] & =\mathbf{W}_{\text {out }} \mathbf{x}_{\text {detector }}[k] .
\end{aligned}
$$

Equation (1) is the reservoir state update equation, where $\mathbf{x}$ is the state of the reservoir; $f$ is a nonlinear vector field to account for the nonlinear behaviour of the reservoir nodes and $\mathbf{u}$ is the input signal to the reservoir. $u_{\text {bias }}$ is a bias signal applied to the nodes of the reservoir, that can be non-zero in the case of NRZ signals, or - as in our case - zero for RZ signals. $\mathbf{W}_{\text {res }}$ is the interconnection matrix, that represents the connections between the nodes of the reservoir, and taking into account splitting ratios, losses, and random phase shifts uniformly distributed on $[-\pi, \pi]$. Finally, $\mathbf{W}_{\text {in }}$ is the input matrix, representing the input weight on each node. In our architecture, we inject the same power modulation in all the active nodes with random phase shifts, hence $\mathbf{W}_{\text {in }}$ is a $16 \times 16$ diagonal matrix with random elements sampled from a uniform distribution over the interval $[-\pi, \pi]$.

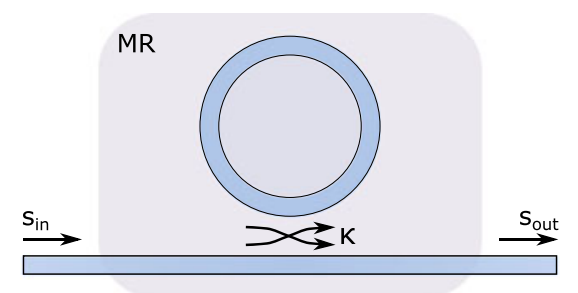

Fig. 2. Illustration of a SOI microring resonator as a nonlineaer node of the reservoir. The node is a two-ports photonics component integrated on silicon.

Equation (2) gives the output $\mathbf{y}_{\text {out }}$ of the reservoir. $\mathbf{x}_{\text {detector }}$ are the states of the reservoir after the detectors, and $\mathbf{W}_{\text {out }}$ is the readout matrix comprising the output weights that need to be determined through training by a ridge regression.

The detector used in our simulations is the same as the one used in previous work [23] and its model is based on the Alphalas UPD-15-IR2-FC photodetector. This takes into account the bandwidth limitation of the detector (modelled by a low-pass filter with a $3 \mathrm{~dB}$ cutoff), the response-time limitations, the responsivity, and various noise contributions, including shot noise and thermal noise. The total noise $\sigma_{n}^{2}$ is given by (3):

$$
\sigma_{n}^{2}=2 q B\left(\langle I\rangle+\left\langle I_{d}\right\rangle\right)+\frac{4 k_{B} T B}{R_{L}},
$$

where $B$ is the bandwidth $(B=25 \mathrm{GHz}),\langle I\rangle$ and $\left\langle I_{d}\right\rangle$ are respectively the mean value of the photocurrent and the dark current $\left(\left\langle I_{d}\right\rangle=0.1 \mathrm{nA}\right), q$ is the elementary particle charge, $k_{B}$ is Boltzmann's constant, $T$ is the temperature (in $\mathrm{K}$ ), and $R_{L}$ is the load impedance $\left(R_{L}=50 \Omega\right)$. The mean value of the photocurrent is calculated from $\langle I\rangle=r \cdot N E P \cdot \sqrt{B}$ and the values given in the datasheet of the photodetector: the responsivity $r=0.5 \mathrm{~A} / \mathrm{W}$, and the noise equivalent power $\left(N E P=10^{-15} \mathrm{~W} / \sqrt{\mathrm{Hz}}\right)$.

\section{SINGLE NODE OF THE RESERVOIR}

\section{A. Nonlinear Microring Resonator}

We present in this section the detailed model of a nonlinear microring resonator, used as one of the building blocks of our reservoir architecture, and shown in Fig. 2. The theoretical framework we use is based on the well-established coupledmode theory (CMT). The model described in our work has already been proposed and was able to correctly describe for the SOI microrings a wide range of dynamical behaviors observed experimentally [25], [26].

The input/output relation is given in (4), in which $s_{\text {in }}$ is the input signal (with $P_{\text {in }}=\left|s_{\text {in }}\right|^{2}$ the input power), $s_{\text {out }}$ the output signal (with $P_{\text {out }}=\left|s_{\text {out }}\right|^{2}$ the output power), $\phi_{c}$ the phase propagation in the bus waveguide, $\kappa$ the coupling between the bus waveguide and the microring, and $a$ the complex amplitude of the optical mode in the cavity (with $|a|^{2}$ the energy in the cavity).

$$
s_{\mathrm{out}}=e^{j \phi_{c}} s_{\mathrm{in}}+\kappa a .
$$

The state variables of the SOI nonlinear microring resonator within the CMT-framework are: $a$ the complex amplitude of 
the optical mode, $\Delta T$ the mode-averaged temperature difference between the circular waveguide of the microring and its surroundings, and $N$ the number of free carriers. These variables account for the physical effects taking place in a nonlinear microring resonator: specifically, (i) the two-photon absorption (TPA), which generates free carriers; (ii) the free carrier absorption (FCA) (i.e.) absorption of light by the free carriers; (iii) the free carrier dispersion (FCD) and (iv) losses.

The nonlinear dynamical equations controlling the temporal evolution of the three state variables are given in (5)-(7), with typical time scales $\tau_{a} \approx 21 \mathrm{ps}, \tau_{t h}=65 \mathrm{~ns}$, and $\tau_{f c}=5.3 \mathrm{~ns}$.

$$
\begin{aligned}
\frac{\mathrm{d} a}{\mathrm{~d} t} & =\left[j\left(\omega_{r}+\delta \omega_{n l}-\omega\right)-\frac{\gamma_{\text {loss }}}{2}\right] a+\kappa s_{\mathrm{in}}, \\
\frac{\mathrm{d} \Delta T}{\mathrm{~d} t} & =-\frac{\Delta T}{\tau_{t h}}+\frac{\Gamma_{t h} \gamma_{a b s}|a|^{2}}{\rho_{S i} c_{p, S i} V_{t h}}, \\
\frac{\mathrm{d} N}{\mathrm{~d} t} & =-\frac{N}{\tau_{f c}}+\frac{\Gamma_{F C A} \beta_{S i} c^{2}|a|^{4}}{2 \hbar \omega V_{F C A}^{2} n_{g}^{2}},
\end{aligned}
$$

where $\omega=2 \pi c / \lambda$ and $\omega_{r}=2 \pi c / \lambda_{r}$ with $\lambda_{r}=1552.770 \mathrm{~nm}$ are the frequency of the input light and the resonance frequency of the ring, respectively. The relaxation times for the temperature variations and the free carriers are respectively given by $\tau_{t h}$ and $\tau_{f c}$. TPA in silicon is governed by the constant $\beta_{S i}$. $n_{S i}, c_{p, S i}$, and $\rho_{S i}$, which are the refractive index, the thermal capacity, and the density of the bulk silicon, respectively. We neglect dispersion, thus the group index $n_{g}$ is equal to $n_{S i}$. We also define the effective volumes and confinements for each nonlinear effect: $V_{F C A}, \Gamma_{F C A}, V_{T P A}$, and $\Gamma_{T P A}$.

Losses also play an important role, as they introduce coupling between the three state variables. The total loss $\gamma_{\text {loss }}$ results from the sum of absorption losses $\gamma_{a b s}$, coupling losses into the waveguide $\gamma_{\text {coup }}$ (with $\kappa=j \sqrt{\gamma_{\text {coup }} e^{j \phi_{c}}}$ ), and radiation losses $\gamma_{\text {rad }}$. The absorption losses in the ring are due to linear surface absorption, TPA, and FCA, as presented in (8):

$$
\gamma_{a b s}=\gamma_{a b s, l i n}+\Gamma_{T P A} \frac{\beta_{S i} c^{2}|a|^{2}}{n_{g}^{2} V_{T P A}}+\Gamma_{F C A} \frac{\sigma_{S i} c}{n_{g}} N,
$$

where $\gamma_{a b s, l i n}$ is the linear absorption constant, and $\sigma_{S i}$ is the absorption cross section of FCA in silicon. In the case of a critically coupled ring, we also have $\gamma_{\text {coup }}=\gamma_{a b s, l i n}+\gamma_{\text {rad }}$.

Finally, we give in (9) the expression of the nonlinear detuning $\delta \omega_{n l}$, that is caused by the thermo-optic effect and FCD, while the Kerr-effect is here neglected:

$$
\delta \omega_{n l}=-\frac{\omega_{r}}{n_{g}}\left(\frac{\mathrm{d} n_{S i}}{\mathrm{~d} T} \Delta T+\frac{\mathrm{d} n_{S i}}{\mathrm{~d} N} N\right) .
$$

As for any optical injection study, the two parameters of interest are the input power $P_{\text {in }}=\left|s_{\text {in }}\right|^{2}$, and the wavelength difference between the injected light and the resonance wavelength of the nonlinear microring resonator, that is the optical detuning $\delta \lambda=\lambda-\lambda_{r}$. For the other parameters of the model, we use the typical numerical values listed in Table I [25], [26]. These values will be later used in all our numerical simulations.
TABLE I

Parameters Values Used in the Simulations of the Microring MODEL, ADAPTED FROM [25] AND [26]

\begin{tabular}{ll}
\hline \hline Parameter & Value \\
\hline$\beta_{S i}$ & $8.4 \times 10^{-12} \mathrm{~m} \cdot \mathrm{W}^{-1}$ \\
$\mathrm{~d} n_{s i} / \mathrm{d} T$ & $1.86 \times 10^{-4} \mathrm{~K}-1$ \\
$\mathrm{~d} n_{s i} / \mathrm{d} N$ & $-1.73 \times 10^{-27} \mathrm{~m}^{3}$ \\
$\sigma_{S i}$ & $10^{-21} \mathrm{~m}^{2}$ \\
$\rho_{S i}$ & $2.33 \mathrm{~g} \cdot \mathrm{cm}^{-3}$ \\
$c_{p, S i}$ & $0.7 \mathrm{~J} \cdot \mathrm{g}^{-1} \cdot \mathrm{K}^{-1}$ \\
$n_{g}=n_{S i}$ & 3.476 \\
$\eta_{l i n}$ & 0.4 \\
$\gamma_{a b s, l i n}$ & $2 \eta_{l i n} / 205 \mathrm{ps}^{-1}$ \\
$\gamma_{\text {coup }}$ & $2 / 205 \mathrm{ps}^{-1}$ \\
$\tau_{t h}$ & $65 \mathrm{~ns}$ \\
$\tau_{f c}$ & $5.3 \mathrm{~ns}$ \\
$\Gamma_{t h}$ & 0.9355 \\
$\Gamma_{T P A}$ & 0.9964 \\
$\Gamma_{F C A}$ & 0.9996 \\
$V_{t h}$ & $3.19 \mu \mathrm{m}^{3}$ \\
$V_{T P A}$ & $2.59 \mu \mathrm{m}^{3}$ \\
$V_{F C A}$ & $2.36 \mu \mathrm{m}^{3}$ \\
\hline
\end{tabular}

\section{OPERATING Point OF THE RESERVOIR}

It is necessary to choose an operating point of the reservoir to achieve a good level of performance to solve complex tasks. It was demonstrated that an adequate operating point for a reservoir is a fixed point, close to instabilities in order to maximize the complexity of the transient to the steady-state [37]. We make the simple assumption that the reservoir will be in a steady state if a single node of the reservoir is on a fixed point. This is a reasonable assumption because of the weak linear optical coupling due to the losses induced by the waveguides $(3 \mathrm{~dB} / \mathrm{cm})$, the splitters ( $3 \mathrm{~dB}$ for each splitter), and the combiners ( $3 \mathrm{~dB}$ for each combiner).

Hence, we first simulate a single, uncoupled, nonlinear microring resonator subjected to steps of optical power between $P_{\mathrm{in}_{0}}=0 \mathrm{~mW}$ and several maximum values $P_{\mathrm{in}_{1}}$. The simulations are performed as follow: we integrate the CMT-model of the nonlinear microring resonator (see [25] and [26] for the equations and the parameters values) over $2.5 \mu \mathrm{s}$ with a power step from $P_{\mathrm{in}_{0}}=0 \mathrm{~mW}$ to the value of $P_{\mathrm{in}_{1}}$ at $t=100 \mathrm{~ns}$. We use an Euler integration method with a $1.0 \mathrm{ps}$ integration time step, and a $10.0 \mathrm{ps}$ sampling time. These simulations are performed using the Caphe software environment [38].

We then extract from the time series the consecutive extrema for each value of the maximum input power, after deleting the transients. We plot the extrema for each value of the maximum input power at different values of the optical detuning, and obtain the bifurcation diagrams shown in Fig. 3(a)-(c), for respectively (a) $\delta \lambda=0 \mathrm{pm}$, (b) $\delta \lambda=-50 \mathrm{pm}$, and (c) $\delta \lambda=50 \mathrm{pm}$.

Fig. 3(a) shows the output power of a microring resonator with an optical detuning $\delta \lambda=0 \mathrm{pm}$, which is a fixed point for $P_{\mathrm{in}_{1}}<0.52 \mathrm{~mW}$, and a self-pulsation (SP) for $P_{\mathrm{in}_{1}}>0.54 \mathrm{~mW}$. For an optical detuning $\delta \lambda=-50 \mathrm{pm}$ (see Fig. 3(b)), the output power is always a fixed point for $P_{\mathrm{in}_{1}}<2.0 \mathrm{~mW}$. Finally, we see in Fig. 3(c) that, for an optical detuning $\delta \lambda=50 \mathrm{pm}$, the output power is stationary for $P_{\mathrm{in}_{1}}<0.38 \mathrm{~mW}$, and a self-pulsating for $P_{\mathrm{in}_{1}}>0.40 \mathrm{~mW}$. 

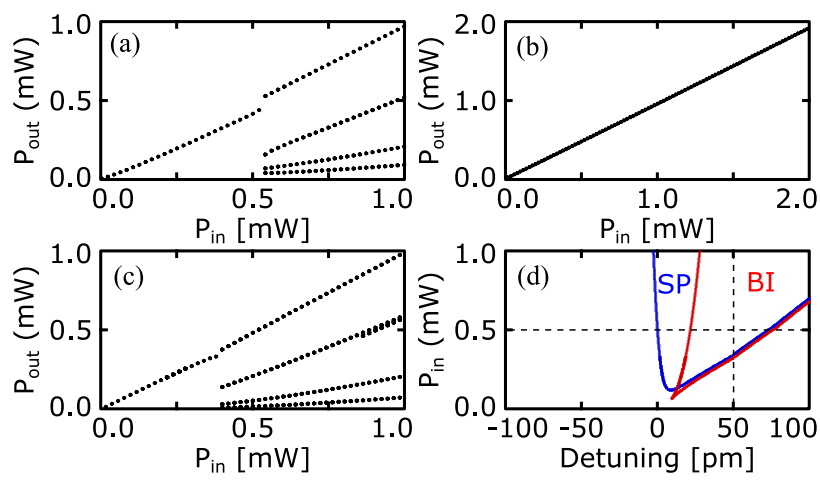

Fig. 3. (a)-(c) Bifurcation diagrams of a single nonlinear microring resonator The bifurcation parameter is the injected power $P_{\text {in }}$ (in $\mathrm{mW}$ ), and we give the diagrams for various values of the optical detuning. (a) $\delta \lambda=0 \mathrm{pm}$, (b) $\delta \lambda=-50 \mathrm{pm}$, and (c) $\delta \lambda=50 \mathrm{pm}$. (d) Stability map of a nonlinear microring resonator in the $\left(\delta \lambda, P_{\text {in }}\right)$ plane. Figure adapted from [25] and [26], using continuation techniques.

From these bifurcation diagrams, we identify an operating point for the reservoir in terms of power amplitude modulation, for each value of the optical detuning. For $\delta \lambda=0 \mathrm{pm}$, we choose $P_{\mathrm{in}_{1}}=0.5 \mathrm{~mW}$ (close to the SP bifurcation point). For $\delta \lambda=-50 \mathrm{pm}$, any value of the injected power is possible, but we also choose $P_{\mathrm{in}_{1}}=0.5 \mathrm{~mW}$ to guarantee low levels of energy consumption. Finally, for $\delta \lambda=50 \mathrm{pm}$, we choose $P_{\mathrm{in}_{1}}=0.3 \mathrm{~mW}$.

Finally, in Fig. 3(d), we present a theoretically obtained stability map of a nonlinear microring resonator. This shows the ring's dynamical behavior in the optical detuning/injected power plane, and for a given set of injection parameters. We find three different regions associated to stable fixed points, self-pulsing, and bistability when two different states can be reached depending of the initial conditions. Each region is delimited by bifurcation points: two saddle-node and a supercritical Hopf bifurcation for the bistable and self-pulsing region, respectively. Note that this map was originally presented in a normalized parameter plane [25], [26], but we have recomputed it with continuation techniques and reformatted it with respect to our parameters of interest.

With Fig. 3(d), it is possible to extract the information of Fig. 3(a)-(c) for any optical detuning; thus finding the value of injected power for which the microring is on a fixed point close to self-pulsing. This allows to set an optimal operating parameter conditions for the reservoir.

\section{Numerical Simulations: Methods \& Performance}

\section{A. Simulation Methods}

We obtain the reservoir states through the simulation, using the Caphe photonic circuit simulator [38], of the $4 \times 4$ (16 nodes) swirl reservoir, described by (1) and (2), using nonlinear microring resonators as nodes.

The performance of the reservoir is measured on the delayed XOR task, as defined in (10). The current output bit $y[n]$ for this task is the Boolean XOR operation between the current input bit $x[n]$ with the bit that is $n_{\text {delay }}$ bits in the past $x\left[n-n_{\text {delay }}\right]$. This task is considered as the most difficult two-bits binary delayed task, due to the nonlinear separability in machine learning terms [23]. In our simulations, we always assume $n_{\text {delay }}=1$.

$$
y[n]=x[n] \oplus x\left[n-n_{\text {delay }}\right] .
$$

The bit stream fed into the reservoir consists of 20000 randomly chosen bits. The training of the linear readouts is performed using regularized ridge regression on 16000 bits, using the scikit-learn library [39]. The testing is done on the 4000 remaining bits, for a regularization parameter chosen using the best case from a five-fold cross-validation. We report the error rates on the test data, hence the minimum measurable error rate is $2.5 \times 10^{-4}$. Multiple-input simulations are performed with the same bit stream injected simultaneously with the same input power weights on all 16-nodes. For the readout layer, we also use the discrete states $x_{\text {detector }}$ of all 16-nodes to perform the training and the testing of the reservoir. We use in all our performance simulations a sampling rate of $160 \mathrm{~Gb} / \mathrm{s}$.

In this work, we investigate the optimal design of the reservoir in terms of interconnection lengths, for a fixed data rate. Hence, we will plot the reservoir performance as a function of the reservoir inter-delay, that is the time the light needs to travel in the waveguide from one node to the next. The length $L$ of the bus waveguide between two consecutive nodes can then be obtained through (11), where $n_{S i}=3.476$ is the refractive index of the bulk silicon, and $t_{\text {delay }}$ is the reservoir inter-delay.

$$
L=\frac{c \times t_{\text {delay }}}{n_{S i}} .
$$

The section is organised as follows: we present the performance of the 16-nodes reservoir when focusing alternatively on the influence of the bit rate, the optical detuning and the power modulation.

\section{B. Performance: Influence of the Bit Rate}

The reservoir performance is plotted in Fig. 4. We focus on the influence of the data rate, and give the performance as a function of the reservoir inter-delay at $10 \mathrm{~Gb} / \mathrm{s}$ (black dots), $15 \mathrm{~Gb} / \mathrm{s}$ (red squares), $20 \mathrm{~Gb} / \mathrm{s}$ (blue triangles), and $30 \mathrm{~Gb} / \mathrm{s}$ (green diamonds), respectively. In order to compare with previous work, we give in Fig. 4(a) the performance of the fully passive reservoir of [22] and [23], and in Fig. 4(b) the performance of the reservoir using nonlinear microring resonators as nodes (called MR-reservoir for clarity purposes).

In the case of the passive reservoir (Fig. 4(a)), the bit stream is fed on all nodes through a power modulation from $P_{\mathrm{in}_{0}}=$ $0.1 \mathrm{~mW}$ and $P_{\mathrm{in}_{1}}=0.2 \mathrm{~mW}$. In the MR-reservoir (Fig. 4(b)), we fix the optical detuning at $\delta \lambda=50.0 \mathrm{pm}$, and we modulate the injected power between $P_{\mathrm{in}_{0}}=0.0 \mathrm{~mW}$ and $P_{\mathrm{in}_{1}}=0.3 \mathrm{~mW}$, according to the optimal injection parameter conditions determined previously. In this reservoir, all microrings have the same resonance frequency, and we have used the photodetector model previously described.

The results presented in this figure suggest that the reservoir with nonlinear microrings as nodes can perform the typical delayed XOR task with error rates about $2.5 \times 10^{-4}$ (lowest achievable value with the number of bit used in testing) for 

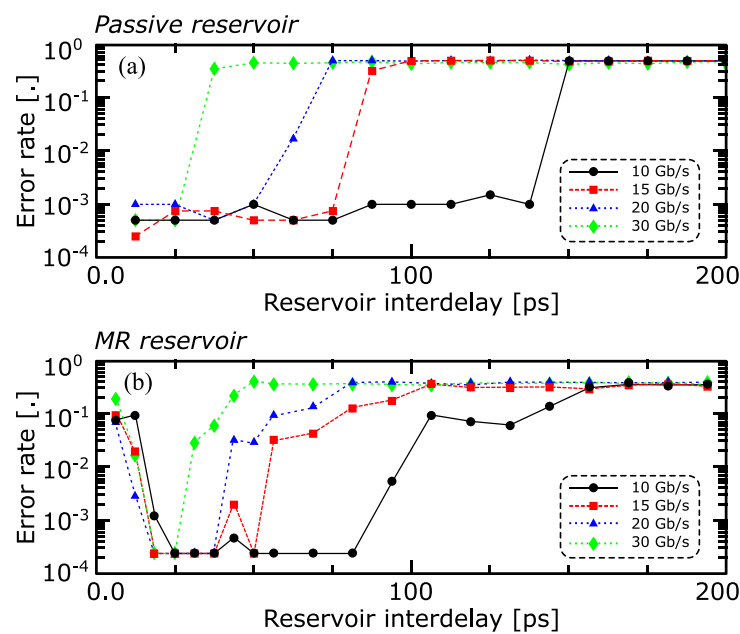

Fig. 4. Error rate - for the XOR task - as a function of the reservoir interdelay for various bit rates: $10 \mathrm{~Gb} / \mathrm{s}$ (black dots), $15 \mathrm{~Gb} / \mathrm{s}$ (red squares), $20 \mathrm{~Gb} / \mathrm{s}$ (blue triangles), and $30 \mathrm{~Gb} / \mathrm{s}$ (green diamonds). Comparison between (a) the passive reservoir of [22] and [23], and (b) the dynamically active reservoir. (a) We modulate the injected power between $P_{\mathrm{in}_{0}}=0.1 \mathrm{~mW}$ and $P_{\mathrm{in}_{1}}=0.2 \mathrm{~mW}$. (b) The optical detuning is $\delta \lambda=50.0 \mathrm{pm}$, and we modulate the injected power between $P_{\mathrm{in}_{0}}=0.0 \mathrm{~mW}$ and $P_{\mathrm{in}_{1}}=0.3 \mathrm{~mW}$. The minimum acceptable error rate is $2.5 \times 10^{-4}$.
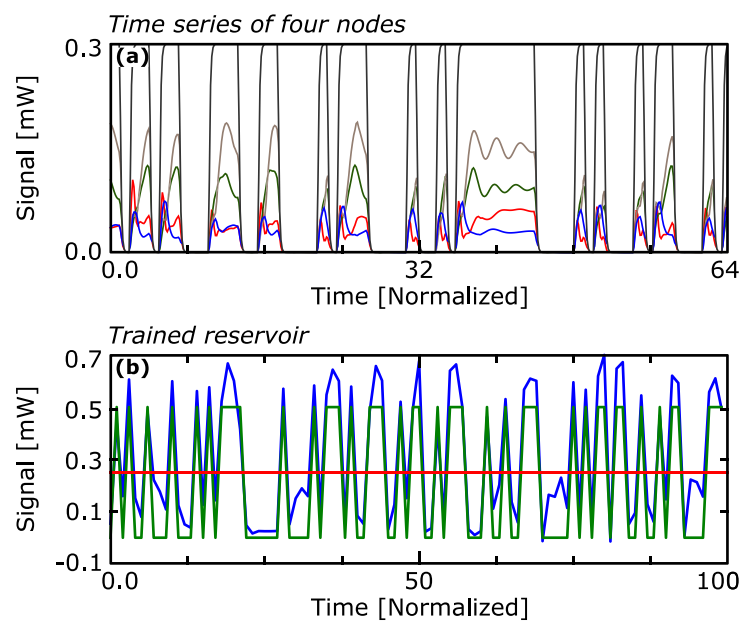

Fig. 5. (a) Time series of four different nodes of the reservoir for the following injection conditions (black line): an optical detuning $\delta \lambda=50.0 \mathrm{pm}$, a power modulation between $P_{\mathrm{in}_{0}}=0.0 \mathrm{~mW}$ and $P_{\mathrm{in}_{1}}=0.3 \mathrm{~mW}$ at $20 \mathrm{~Gb} / \mathrm{s}$, and an inter-delay $t_{\text {interdelay }}=18.75$ ps. (b) Desired output (green curve), trained output of the reservoir (blue curve), and decision threshold (red line) for the same injection parameters. These parameters correspond to an optimal value of the error rate of Fig. 4.

various values of the inter-delay at high bit rates. We also see that the range of inter-delay values, where the reservoir performs at its best, is slightly greater for lower bit rates. This is similar to the passive reservoir (Fig. 4), but our architecture can achieve lower error rates. We notice also a reduced range of inter-delay values for the best performance compared to a passive reservoir. This is most likely due to the internal time scale of the optical mode $\tau_{a} \approx 20 \mathrm{ps}$ in the microring resonator model. This time scale is close to the optimal value of inter-delay in term of reservoir performance.

We present also in Fig. 5(a) normalized time series generated by four nodes of the reservoir, along with the input power in each node. These time series are obtained for the simulation
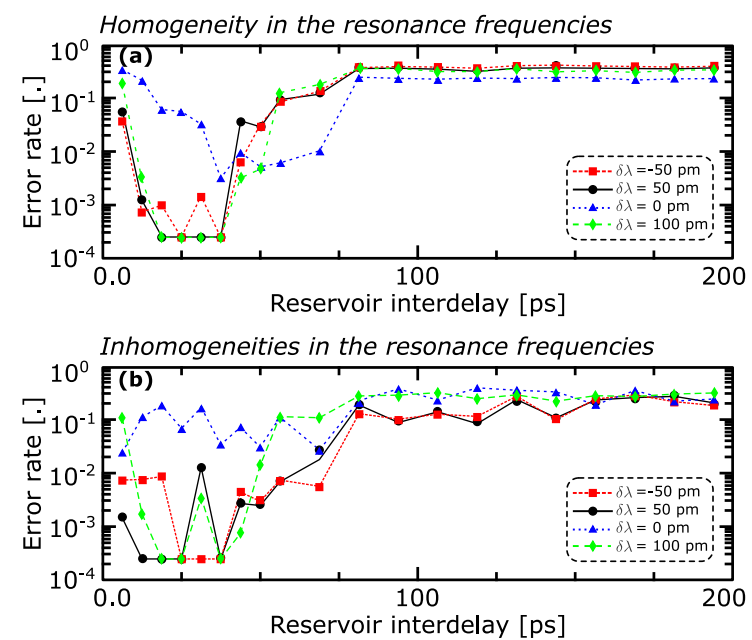

Fig. 6. Error rate - for the XOR task - as a function of the reservoir inter-delay for various values of the optical detuning at $20 \mathrm{~Gb} / \mathrm{s}$. The power modulation is chosen so that a microring alone is in a stationary state, but close to the instabilities, with $P_{\mathrm{in}_{0}}=0.0 \mathrm{~mW} . \delta \lambda=-50 \mathrm{pm}$ and $P_{\mathrm{in}_{1}}=0.5 \mathrm{~mW}$ (red squares), $\delta \lambda=0.0 \mathrm{pm}$ and $P_{\mathrm{in}_{1}}=0.5 \mathrm{~mW}$ (blue triangles), $\delta \lambda=50 \mathrm{pm}$ and $P_{\mathrm{in}_{1}}=0.3 \mathrm{~mW}$ (black dots), and $\delta \lambda=100 \mathrm{pm}$ and $P_{\mathrm{in}_{1}}=0.5 \mathrm{~mW}$ (green diamonds). In (a), the microrings are all identical, and in (b), each microring has a different value of the resonance frequency. The minimum acceptable error rate is $2.5 \times 10^{-4}$.

of the MR-reservoir for an optical detuning $\delta \lambda=50.0 \mathrm{pm}$, a power modulation comprised between $P_{\mathrm{in}_{0}}=0.0 \mathrm{~mW}$ and $P_{\mathrm{in}_{1}}=0.3 \mathrm{~mW}$, and an inter-delay $t_{\text {interdelay }}=18.75 \mathrm{ps}$. This injection point corresponds to a optimal of the error rate in Fig. 4(b). Finally, in Fig. 5(b), we show the output of the trained reservoir for the same injection parameters as in Fig. 5(a). The green curve is the desired output, the blue curve is the output of the trained reservoir, and the red line is the decision threshold. For both Fig. 4(a) and (b), the time is normalized so that one bit is equal to one unit of time.

\section{Performance: Influence of the Optical Detuning}

In this part, we focus on the influence of the optical detuning on the MR-reservoir performance. Fig. 6(a) gives the performance of the reservoir as a function of the inter-delay for four different values of the optical detuning: $\delta \lambda=-50 \mathrm{pm}$ (red squares), $\delta \lambda=0.0 \mathrm{pm}$ (blue triangles), $\delta \lambda=50 \mathrm{pm}$ (black dots), and $\delta \lambda=100 \mathrm{pm}$ (green diamonds). The RZ power modulation is chosen so that a microring alone is in a stationary state, but close to a bifurcation point. Referring to Fig. 3(a)-(c), the high value of the power modulation is $P_{\mathrm{in}_{1}}=0.3 \mathrm{~mW}$ for $\delta \lambda=50 \mathrm{pm}$, and $P_{\mathrm{in}_{1}}=0.5 \mathrm{~mW}$ for $\delta \lambda=0 \mathrm{pm}, \delta \lambda=100 \mathrm{pm}$, and $\delta \lambda=-50 \mathrm{pm}$.

In this figure, we have considered that the microrings are strictly the same, meaning that all 16 nonlinear microring resonators have the same resonance frequency. For more realistic simulations, we give in Fig. 6(b) - and for the same input conditions - the performance of the MR-reservoir when the resonance frequencies of the microrings are different. The resonance frequencies of the 16 microring resonators follow a Gaussian distribution centred on respectively $\delta \lambda \in\{-50,0.0,50,100\} \mathrm{pm}$, with a $10 \mathrm{pm}$ standard deviation, that is a rather pessimistic value with regard to the current technology. 


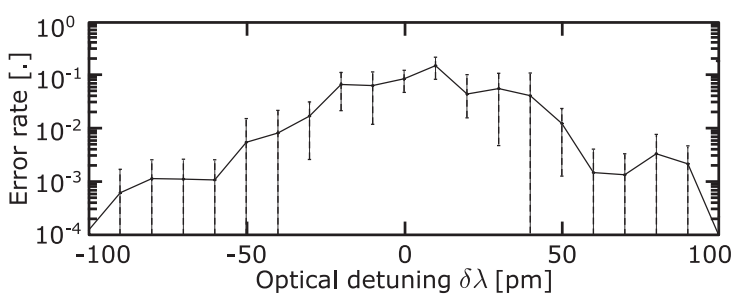

Fig. 7. Error rate - for the XOR task - as a function of the optical detuning for a power modulation between $P_{\mathrm{in}_{0}}=0.0 \mathrm{~mW}$ and $P_{\mathrm{in}_{1}}=0.5 \mathrm{~mW}$, an interdelay of $18.75 \mathrm{ps}$, and a bitrate $20 \mathrm{~Gb} / \mathrm{s}$. Error bars are given for seven series of simulations. The minimum acceptable error rate is $2.5 \times 10^{-4}$.

Fig. 6 shows that the reservoir performs better when the value of the optical frequency of the injected light is detuned with respect to the resonance frequency of the nonlinear node (typically in our study $\delta \lambda \in\{-50,50,100\} \mathrm{pm}$ ), than when the light is injected at the resonance frequency of the microring resonator (i.e. $\delta \lambda=0 \mathrm{pm}$ ). Note that the performance is similar for those three different values of the optical detuning $(\delta \lambda \in\{-50,50,100\} \mathrm{pm})$. Intuitively, this can be understood by the filtering properties of microrings: they absorb more optical power if the frequency of the injected light is close to their resonance. As a result, the wave-mixing between the nodes in the network is reduced, thus impeding the reservoir computer performance. Fig. 6(b) shows a good robustness of the reservoir with regards to heterogeneities in the frequency resonance between the nodes, providing that the detuning of the injected light is larger than the standard deviation of the heterogeneities in resonance.

In order to corroborate the results of Fig. 6, we plot in Fig. 7 the performance of the reservoir as a function of the optical detuning. More specifically, we have set the inter-delay (18.75 ps), the power modulation between $P_{\mathrm{in}_{0}}=0.0 \mathrm{~mW}$ and $P_{\mathrm{in}_{1}}=0.5 \mathrm{~mW}$, and we have followed the horizontal dashed line of Fig. 3(d). This value of inter-delay corresponds to the best choice in terms of interconnection length, as it ensures relatively small connection waveguides, while the mismatches in the frequency resonance of the rings does not affect the performance of the reservoir (see Fig. 6(b)). Similarly to Fig. 6(b), we have introduced mismatches in the resonance frequency between the rings. We realize seven experiments for each optical detuning; the results are averaged and we give the error bars.

Fig. 7 unveils a better level of performance when the frequency of the injected light is far from the frequency resonance of the nonlinear microring resonators. We see also that the performance are better for negative values of the frequency detuning. This can be understood by looking at the stability map of Fig. 3(d), and the horizontal dashed line that we have followed. For positive values of the optical detuning from $0 \mathrm{pm}$ and $75 \mathrm{pm}$, a nonlinear microring resonator is self-pulsing for an injected power of $0.5 \mathrm{~mW}$, thus meaning the reservoir is not on a steady state and consequently reducing its performance.

\section{Performance: Influence of the Injection Power}

In this section, we focus on the power budget considerations. More specifically, we fix the optical detuning between

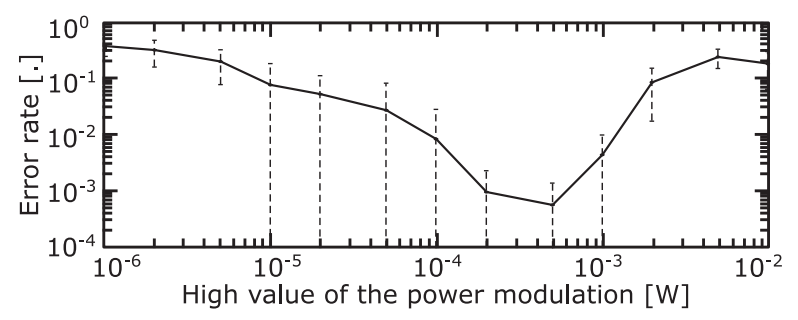

Fig. 8. Error rate - for the XOR task - as a function of the high value of the power modulation for an optical detuning $\delta \lambda=50 \mathrm{pm}$, an inter-delay of $18.75 \mathrm{ps}$, and a bitrate $20 \mathrm{~Gb} / \mathrm{s}$. The low value of the power modulation is $P_{\mathrm{in}_{0}}=0.0 \mathrm{~mW}$. Error bars are given for seven series of simulations. The minimum acceptable error rate is $2.5 \times 10^{-4}$.

the injected light and the resonance frequency of the rings $(\delta \lambda=50 \mathrm{pm})$, we fix again the interconnection delay at $18.75 \mathrm{ps}$, and we plot the performance of the reservoir for various values of the power modulation. This is always a return-to-zero (RZ) modulation, and we plot the error rate as a function of the high value of the power modulation, following the vertical dashed line of Fig. 3(d). Note that we have also introduced mismatches in the resonance frequency between the rings, similarly to previous studies. We give the average and the error bars for seven series of simulations.

Fig. 8 shows that values of the injected power lower than $10^{-4} \mathrm{~W}$ result in a degradation of the performance, due to a reduction of total power on each node and wave-mixing between the nodes through losses in the other integrated elements (waveguides, splitters, combiners). We also see that the performance of the reservoir are low for high values of the modulation $\left(P_{\mathrm{in}_{1}}>10^{-3} \mathrm{~W}\right)$, due to the fact that each node is self-pulsing for these injection parameters (see stability map, Fig. 3(d)). Finally, the optimal operating condition at this particular optical detuning is when the high value of the power modulation leads a single microring resonator to be in a steady state, but close to instabilities. However, we find a very good performance obtained for the high value of the power modulation $P_{\mathrm{in}_{1}}=0.5 \times 10^{-3} \mathrm{~W}$, where a single microring resonator alone is self-pulsing.

In the previous sections, we have perform most of our simulations at $\delta \lambda=50 \mathrm{pm}$, with a power modulation from $P_{\mathrm{in}_{0}}=0.0 \mathrm{~W}$ to $P_{\mathrm{in}_{1}}=0.3 \mathrm{~mW}$, which is in the interval of best performance at this particular detuning. Hence the total power budget is very low. Indeed the chip is only powered by the optical power using the same bit stream input on each node, thus the averaged power needed for the reservoir to perform is $N_{\text {nodes }} \times 0.5 \times\left(P_{\text {in }_{1}}-P_{\text {in }_{0}}\right)=2.4 \mathrm{~mW}$ (with $N_{\text {nodes }}=16$, the number of nodes in the reservoir). Moreover, unlike the purely passive reservoir, where a bias power was necessary to perform optimally, the MR-reservoir has the best performance when there is no power bias, thus reducing the mean power consumption.

\section{CONCLUSION}

To conclude, we have suggested a novel integrated reservoir architecture using microring resonators as nonlinear nodes, that can perform at state-of-the-art level of performance on a nonlin- 
ear Boolean task for various operating parameter conditions. We also have connected the performance of the reservoir computer with the nonlinear properties of the nodes stability with respect to injected power and frequency detuning between the injected light and the resonance of the rings.

More specifically, we have studied the influence of the data rate, and shown that the intrinsic presence of three distinct time scales in the model of the nonlinear nodes leads to the need to carefully design the reservoir in terms of the length of the interconnections between the nodes. We have also investigated the influence of two critical operational parameters in the network dynamics: (i) the injected power and (ii) the optical detuning. We have found that a large variety of operating conditions can lead to optimal performance of the reservoir on the typical delayed XOR task, when some important conditions are fulfilled. First, each node should be in a steady state, close to instabilities. This condition, along with a stability map of a single node, allows us to choose the operating condition of the complete reservoir parameters for optimal performance. We have also found a good robustness when we introduce heterogeneities in the properties of the nonlinear nodes, for example in the frequency resonance between the ring resonators.

We have demonstrated that this integrated reservoir can perform very well on a typical boolean task, with very low power consumption. Considering the RZ power modulation between $P_{\mathrm{in}_{0}}=0.0 \mathrm{~mW}$ and $P_{\mathrm{in}_{1}}=0.3 \mathrm{~mW}$ with the same bit stream input on each node, the power budget is very good, and could be further improved in future work by reducing the number of injected nodes, for instance by injecting the data only on the four central nodes, as suggested in previous work by some of the authors [23]. Moreover, from an experimental point of view, it is simpler to inject the data on fewer nodes, as it reduces the routing density on the chip.

Contrary to the passive reservoir of [22], [23] in which the nonlinearity is in the readout ((i.e.) the detector), we have integrated nonlinear elements (the microring resonators) in the recurrence of the network. This work shows that the performance on this particular task in terms of BER and power consumption are very similar with the previous design. This is mainly due to the losses limiting the mixing in both architectures with or without embedded nonlinear elements. A different internal architecture with better losses management would probably enhance our performance in presence of microring resonators. The current results motivate further investigations on the performance of this kind of structure, especially by studying the performance on other tasks such as time series generation, chaos prediction, or nonlinear channel equalization to see if the nonlinearities in the recurrence of the network have a good impact on the performance of the reservoir for these complex tasks [10]. This opens new research venues aiming at integrated, high-speed, energyefficient, all-optical data processing for telecom applications.

\section{REFERENCES}

[1] J. Manyika et al., "Big data: The next frontier for innovation, competition, and productivity," New York, NY, USA: McKinsey Global Inst., 2011.
[2] P. Luciano et al., "A hardware implementation of a brain inspired filter for image processing," IEEE Trans. Nucl. Sci., vol. 64, no. 6, pp. 1374-1381, Jun. 2017.

[3] S. Jung and S. Su Kim, "Hardware implementation of a real-time neural network controller with a DSP and an FPGA for nonlinear systems," IEEE Trans. Ind. Electron., vol. 54, no. 1, pp. 265-271, Feb. 2007.

[4] W. Maass, T. Natschläger, and H. Markram, "Real-time computing without stable states: A new framework for neural computation based on perturbations," Neural Comput., vol. 14, no. 11, pp. 2531-2560, 2002.

[5] H. Jaeger and H. Haas, "Harnessing nonlinearity: Predicting chaotic systems and saving energy in wireless communication," Science, vol. 304, no. 5667 , pp. 78-80, 2004.

[6] M. Lukoševičius and H. Jaeger, "Reservoir computing approaches to recurrent neural network training," Comput. Sci. Rev., vol. 3, no. 3, pp. 127 149, 2009.

[7] F. Schürmann, K. Meier, and J. Schemmel, "Edge of chaos computation in mixed-mode VLSI-A hard liquid," in Proc. Adv. Neural Inf. Process. Syst., 2005, pp. 1201-1208.

[8] D. Verstraeten, B. Schrauwen, and D. Stroobandt, "Reservoir computing with stochastic bitstream neurons," in Proc. 16th Annu. ProRISC Workshop, 2005, pp. 454-459.

[9] M. S. Kulkarni and C. Teuscher, "Memristor-based reservoir computing," in Proc. IEEE/ACM Int. Symp Nanoscale Archit., 2012, pp. 226-232.

[10] G. Van der Sande, D. Brunner, and M. C. Soriano, "Advances in photonic reservoir computing," Nanophotonics, vol. 6, no. 3, pp. 561-576, 2017.

[11] L. Larger et al., "High-speed photonic reservoir computing using a timedelay-based architecture: Million words per second classification," Phys. Rev. $X$, vol. 7, no. 1, 2017, Art. no. 011015.

[12] D. Brunner, M. C. Soriano, C. R. Mirasso, and I. Fischer, "Parallel photonic information processing at gigabyte per second data rates using transient states," Nature Commun., vol. 4, 2013, Art. no. 1364.

[13] L. Appeltant et al., "Information processing using a single dynamical node as complex system," Nature Commun., vol. 2, 2011, Art. no. 468.

[14] F. Duport, B. Schneider, A. Smerieri, M. Haelterman, and S. Massar, "Alloptical reservoir computing," Opt. Express, vol. 20, no. 20, pp. 22783 22795,2012

[15] L. Larger et al., "Photonic information processing beyond turing: An optoelectronic implementation of reservoir computing," Opt. Express, vol. 20, no. 3, pp. 3241-3249, 2012.

[16] M. C. Soriano et al., "Optoelectronic reservoir computing: Tackling noiseinduced performance degradation," Opt. Express, vol. 21, no. 1, pp. 12-20, 2013.

[17] P. Antonik, M. Hermans, F. Duport, M. Haelterman, and S. Massar, "Towards pattern generation and chaotic series prediction with photonic reservoir computers," Proc. SPIE, vol. 9732, 2016, Art. no. 97320B-1

[18] A. N. Tait et al., "Neuromorphic silicon photonics," arXiv:1611.02272, 2016.

[19] K. Vandoorne, J. Dambre, D. Verstraeten, B. Schrauwen, and P. Bienstman, "Parallel reservoir computing using optical amplifiers," IEEE Trans. Neural Netw., vol. 22, no. 9, pp. 1469-1481, Sep. 2011.

[20] C. Mesaritakis, V. Papataxiarhis, and D. Syvridis, "Micro ring resonators as building blocks for an all-optical high-speed reservoir-computing bit-pattern-recognition system," J. Opt. Soc. Amer. B, vol. 30, no. 11, pp. 3048-3055, 2013.

[21] C. Mesaritakis, A. Kapsalis, and D. Syvridis, "All-optical reservoir computing system based on InGaAsP ring resonators for high-speed identification and optical routing in optical networks," in Proc. SPIE, 2015, Art. no. 937033.

[22] K. Vandoorne et al., "Experimental demonstration of reservoir computing on a silicon photonics chip," Nature Commun., vol. 5, 2014, Art. no. 3541.

[23] A. Katumba, M. Freiberger, P. Bienstman, and J. Dambre, "A multipleinput strategy to efficient integrated photonic reservoir computing," Cogn. Comput., vol. 9, pp. 307-314, 2017.

[24] M. A. A. Fiers et al., "Nanophotonic reservoir computing with photonic crystal cavities to generate periodic patterns," IEEE Trans. Neural Netw. Learn. Syst., vol. 25, no. 2, pp. 344-355, Feb. 2014.

[25] T. Van Vaerenbergh, "All-optical spiking neurons integrated on a photonic chip,” Ph.D. dissertation, Dept. Inf. Technol., Dept. Electron. Inf. Syst., Ghent Univ., Ghent, Belgium, 2014.

[26] T. Van Vaerenbergh et al., "Cascadable excitability in microrings," Opt. Express, vol. 20, no. 18, pp. 20292-20308, 2012.

[27] L. Zhang, Y. Fei, Y. Cao, X. Lei, and S. Chen, "Experimental observations of thermo-optical bistability and self-pulsation in silicon microring resonators," J. Opt. Soc. Amer. B, vol. 31, no. 2, pp. 201-206, 2014. 
[28] W. H. Pernice, M. Li, and H. X. Tang, "Time-domain measurement of optical transport in silicon micro-ring resonators," Opt. Express, vol. 18, no. 17 , pp. $18438-18452,2010$.

[29] L. Zhang et al., "Multibistability and self-pulsation in nonlinear high- $Q$ silicon microring resonators considering thermo-optical effect," Phys. Rev. A, vol. 87, no. 5 , 2013, Art. no. 053805 .

[30] S. Chen, L. Zhang, Y. Fei, and T. Cao, "Bistability and self-pulsation phenomena in silicon microring resonators based on nonlinear optical effects," Opt. Express, vol. 20, no. 7, pp. 7454-7468, 2012.

[31] I. S. Amiri, R. Ahsan, A. Shahidinejad, J. Ali, and P. P. Yupapin, "Characterisation of bifurcation and chaos in silicon microring resonator," IET Commun., vol. 6, no. 16, pp. 2671-2675, Nov. 2012.

[32] J. Hryniewicz, P. Absil, B. Little, R. Wilson, and P.-T. Ho, "Higher order filter response in coupled microring resonators," IEEE Photon. Technol. Lett., vol. 12, no. 3, pp. 320-322, Mar. 2000.

[33] H. J. Caulfield, R. A. Soref, and C. S. Vikram, "Universal reconfigurable optical logic with silicon-on-insulator resonant structures," Photon. Nanostruct., Fundam. Appl., vol. 5, no. 1, pp. 14-20, 2007.

[34] H. Kishikawa, T. Kondo, N. Goto, and S. Talabattula, "Optical thresholder consisting of two cascaded Mach-Zehnder interferometers with nonlinear microring resonators," Opt. Eng., vol. 56, no. 8, 2017, Art. no. 086101.

[35] Y. Dumeige, L. Ghisa, and P. Féron, "Integrated all-optical pulse restoration with coupled nonlinear microring resonators," Opt. Lett., vol. 31, no. 14, pp. 2187-2189, 2006.

[36] C. Tanaram et al., "ASK-to-PSK generation based on nonlinear microring resonators coupled to one MZI arm," Procedia Eng., vol. 8, pp. 432-435, 2011.

[37] T. Yamane et al., "Dynamics of reservoir computing at the edge of stability," in Proc. Int. Conf. Neural Inf. Process., 2016, pp. 205-212.

[38] [Online]. Available: http://www.lucedaphotonics.com/. Accessed 2016

[39] [Online]. Available: http://scikit-learn.org/. Accessed 2016.

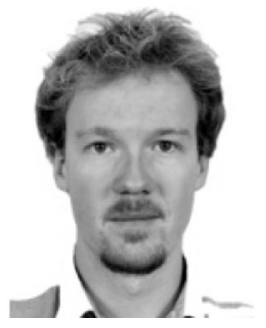

Florian Denis-Le Coarer was born in France, in 1992. He received the Engineering degree from the CentraleSupélec, Metz, France, and the Master's degree from the University of Lorraine, Metz, France, in 2016, both with a major in photonics. He is currently working toward the Ph.D. degree with the CentraleSupélec. His research interests include dynamics of vertical-cavity surface-emitting lasers, and nonlinear dynamics of other photonic systems for neuromorphic applications.

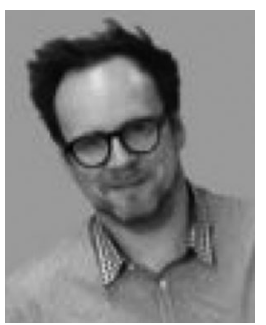

Marc Sciamanna received the Graduate degree in electrical engineering and the Ph.D. degree in applied sciences from the Faculté Polytechnique de Mons, Mons, Belgium, in 2000 and 2004, respectively. In 2009, he received the Habilitation à Diriger les Recherches from the University Paul Verlaine, Metz, France (now the University of Lorraine), in 2012. In 2004, he became an Assistant Professor, and since 2009, he has been a Full Professor with the Supélec, Gif-sur-Yvette, France. He is the Head of the Optics and Electronics Research Group, Supélec, and since 2012, he has been the Adjunct Director of the Laboratoire Matriaux Optiques, Photonique et Systmes, EA 4423. He has authored more than 150 publications, including 60 publications in peer-reviewed journals. His research interests include theory and experiment on nonlinear dynamics of semiconductor lasers with optical injection or optical feedback, optical chaos and chaos synchronization in coupled oscillators, control of optical pattern formation in photorefractive nonlinear cavities, phase-conjugate optical feedback effects, and polarization properties of VCSELs. He was the Chairman and a Committee Member of several international conferences, including SPIE Photonics Europe (2008, 2010, and 2012), the EOS Annual Meeting (2010 and 2012), the IEEE/LEOS Winter Topicals (2009), the IEEE International Semiconductor Laser Conference (2012), PHASE/IPSSO international workshops (2005 and 2007), and A Future in Light International Conference (2009). He is an Associate Editor for the international journal Optical and Quantum Electronics, edited by Springer. He was the recipient of the IEEE Lasers and Electro-Optics Society (now IEEE Photonics Society) in 2002 with a Graduate Student Fellowship Award, the International Society for Optical Engineering in 2003 with the F-MADE Scholarship Award, and the Optical Society of America (OSA) in 2002 with the OSA Newfocus Student Travel Grant. In 2007, he was also the recipient of the TR35 Young Innovator Award from MIT Technology Review (U.S.A.) for his work on controlling chaos in telecom lasers.

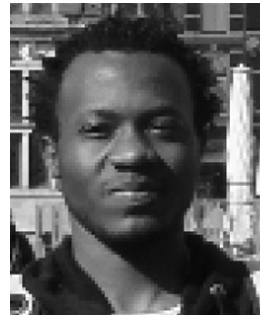

Andrew Katumba was born in Masaka, Uganda, in 1985. He received the M.Sc. degree in optics and photonics from Karlsrule Institute of Technology, Karlsrule, Germany, in 2013. He is currently working toward the Ph.D. degree in photonics engineering with the Photonics Research Group, Ghent University-IMEC, Ghent, Belgium. His current research focuses on photonic neuromorphic architectures for high-speed optical telecommunications systems. He is a student member of the IEEE Photonics Society and the International Society for Optics and Photonics.

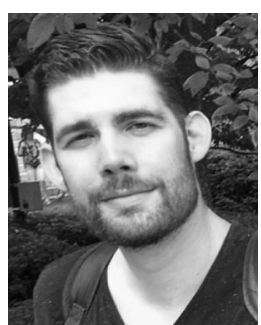

Matthias Freiberger was born in Graz, Austria, in 1983. He received the M.Sc. degree in information and computer engineering from Graz University of Technology, Graz, Austria, in 2016. He is currently working toward the Ph.D. degree with the UGentimec IDLab, Department of Electronics and Information Systems, Ghent University, Ghent, Belgium. His current research focuses on training algorithms for neuromorphic devices. His research interests include scaling up neuromorphic systems, deep learning, and recurrent neural networks on chip.

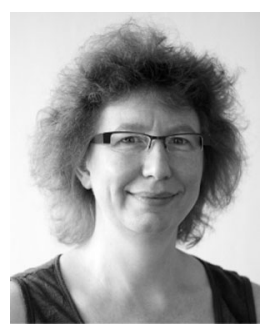

Joni Dambre was born in Ghent, Belgium, in 1973. She received the graduate degree in electrical engineering in 1996 and the Ph.D. degree in computer science engineering in 2003 from the Department of Electronics and Information Systems, Ghent University, Ghent, Belgium. As a Machine Learning Professor, she currently leads an UGent-imec research team (part of IDLab) addressing various topics related to machine learning, artificial and biological neural networks, and neuromorphic hardware.

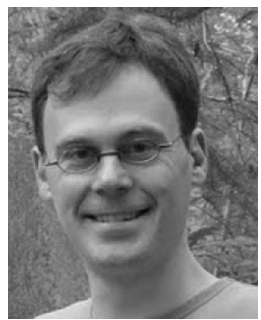

Peter Bienstman was born in Ghent, Belgium, in 1974. He received the graduate degree in electrical engineering in 1997 and the Ph.D. degree in 2001 from the Department of Information Technology, Ghent University, Ghent, Belgium, where he is currently a Full Professor. He has authored and coauthored more than 110 papers and holds several patents. He was the recipient of the ERC starting grant for the Naresco-project: Novel paradigms for massively parallel nanophotonic information processing. His research interests include several applications of nanophotonics (biosensors, photonic information processing, etc.) as well as nanophotonics modeling.

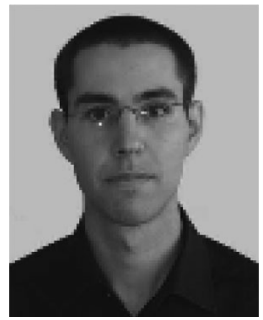

Damien Rontani received the Master of Science degree in electrical engineering (Supélec Curriculum) and the Master's degree in information science, energy, and systems from the Ecole CentraleSupélec, Université Paris-Saclay, Metz, France, in 2004 and 2006, respectively. Meanwhile, he received the Master of Science degree in electrical and computer engineering from Georgia Institute of Technology (Georgia Tech, Atlanta, GA, USA) in 2005. In 2011, he received the $\mathrm{Ph} . \mathrm{D}$. degree in applied physics from the CentraleSupélec and the Ph.D. degree in electrical and computer engineering from Georgia Tech. From 2011 to 2013, he was a Postdoctoral Fellow in the Department of Physics, Duke University, Durham, NC, USA. Since December 2013, he has been an Assistant Professor with the CentraleSupélec, where he conducts leading-edge research in nonlinear dynamics of complex photonics systems, and nonlinear optics for innovative applications in neuroinspired information processing and cognitive computing. His research is conducted within the framework of the OPTEL Research Group and Chair in Photonics, which are parts of the Laboratoire Matriaux Optiques, Photonique et Systmes EA 4423, a joint unit between CentraleSupélec and Université de Lorraine (France). He has authored and coauthored 18 publications in international peer-reviewed journals and more than 45 contributions in international conferences. He was the recipient of the IBM Faculty Award in cognitive computing in 2015 and the JSPS Fellowship for overseas researchers in 2016. 